
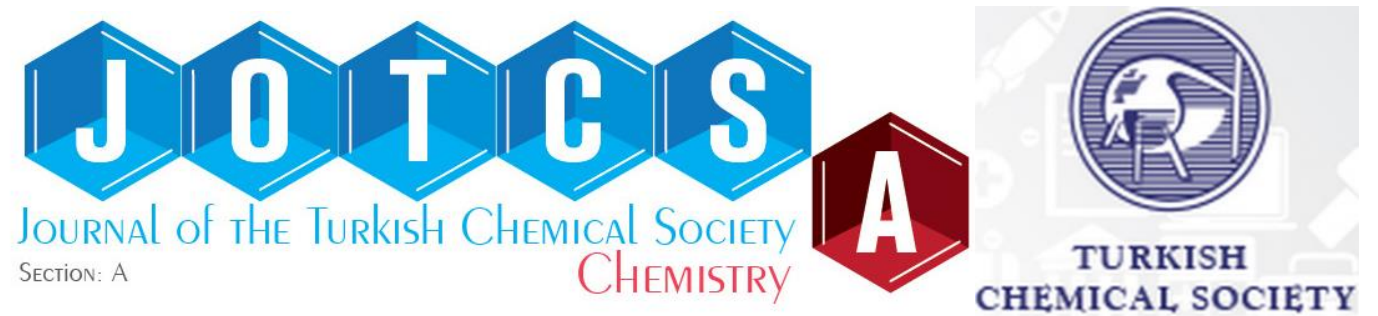

\title{
2-Chlorobenzoylthiourea-modified MCM-41 for Drug Delivery
}

\section{Fatih Mehmet EMEN ${ }^{*} \square$, Ruken Esra DeMİRDöĞEN² KILIÇ}

\author{
${ }^{1}$ Mehmet Akif Ersoy University, Faculty of Arts and Science, Department of Chemistry, TR 15030, Burdur, \\ Turkey \\ ${ }^{2}$ Çankırı Karatekin University, Faculty of Science Department of Chemistry, TR 18100, Çankırı, Turkey \\ 3University of Mersin, Faculty of Science, Department of Chemistry, TR 33100, Mersin, Turkey.
}

Abstract: Mesoporous 2-chlorobenzoylthiourea-modified MCM-41 (2-Cl-BT-MCM-41) was prepared for the first time and loaded with ibuprofen in a supercritical carbon dioxide $\left(\mathrm{SC}-\mathrm{CO}_{2}\right)$ environment. 2-Cl-BT-MCM41 was prepared and also characterized via XRD, FT-IR, SEM and BET techniques. The (100) and (110) reflections observed at low angle XRD show the mesoporous $\mathrm{SiO}_{2}$ structure. The particule size of nonuniform spheres were observed at a range of 250-305 nm. BET surface areas were calculated as $1506 \mathrm{~m}^{2}$ / $\mathrm{g}$ for MCM-41 and $306 \mathrm{~m}^{2} / \mathrm{g}$ for $2-\mathrm{Cl}-\mathrm{BT}-\mathrm{MCM}-41$, respectively. The absorption and releasing studies of ibuprofen were carried out in simulated body fluid. The result revealed that high adsorption capacity for drug with 2-Cl-BT-MCM-41 and slower drug release rate was achieved.

Keywords: Drug delivery system (DDS), mesoporous MCM-41, 2-chlorobenzoylthiourea.

Submitted: October 04, 2018. Accepted: January 18, 2019.

Cite this: Emen F, Demirdöğen R, Avşar G, Kılıç D. 2-Chlorobenzoylthiourea-modified MCM-41 for Drug Delivery. JOTCSA. 2019;6(1):29-34.

DOI: $\underline{\text { https://dx.doi.org/10.18596/jotcsa.467177. }}$

*Corresponding author. E-mail: femen106@gmail.com, Tel: +905077681688, Fax: +902482133099.

\section{INTRODUCTION}

Controlled drug delivery systems (DDS) are formulations that release a drug in a targeted area in the body. Materials obtained by encapsulation of clinically approved drugs will increase the therapeutic effect of the drug and reduce side effects. Biodegradable polymers, polymeric micelles, liposomes, magnetic nanoparticles, hydroxyapatites, calcium phosphate cement (CPC), xerogels, hydrogels and mesoporous silica are used as drug delivery systems. As a disadvantage, hydrolysis leads to deterioration of the polymer-based carrier structure, resulting in a rapid release of drug molecules and non-homogeneous distribution during the separation process. Research is underway to develop inorganic carriers to overcome these disadvantages of polymer systems. Controlled release of drugs from ordering mesoporous materials is an interesting field of application (1). High-order mesoporous silicates are very promising materials with a wide range of possible applications (2). The porous web of these biocompatible materials can act as a reservoir for the maintenance of drug molecules by increasing the solubility and relative bioavailability of drug molecules. Among the mesopoous silicates, the MCM-41 has a high specific surface area ( $\left.1150 \mathrm{~m}^{2} / \mathrm{g}\right)$, a larger pore volume $\left(\sim 1 \mathrm{~cm}^{3} / \mathrm{g}\right)$, and smaller pore size (2-5 $\mathrm{nm}$ ), thus being a drug carrier. MCM-41 has attracted more attention as a carrier for drug delivery (2). MCM has been tested for the transport and controlled release of aspirin (3), atenolol (4), captopril (5), coumarin derivatives (6), diflunisal (7), hydrochlorothazide (8), ibuprofen (9-12), methotrexate (13), naproxine (9), sertraline hydrochloride (14), resveratrol (15), and others (16-17). The presence of high concentrations of silanol group on the silica surface makes it possible to modify the pore walls and surfaces by selecting appropriate organic groups. The surface of MCM-41 can be modified with chloropropyl, phenyl, benzyl, 
mercaptopropyl, cyanopropyl, and aminopropyl groups (18).

The objective of this work was to study the ibuprofen adsorption and release behavior of 2Cl-BT-MCM-41 in simulated body fluid. 2-Cl-BTMCM-41 was prepared and also characterized via XRD, FT-IR, SEM and BET techniques.

\section{MATERIALS AND METHODS}

\section{Synthesis of Compounds Synthesis of MCM-41}

Typically, $0.6 \mathrm{~g}$ of $\mathrm{n}$-cetyltrimethylammonium bromide (CTAB) was first dissolved in $400 \mathrm{~mL}$ of deionized water. Then $3.5 \mathrm{~mL}$ of $2 \mathrm{~mol} / \mathrm{L} \mathrm{NaOH}$ was added to the solution, followed by adjusting the solution temperature to $80{ }^{\circ} \mathrm{C}$. Subsequently, $2.5 \mathrm{~mL}$ of TEOS was added dropwise to the above solution with vigorous stirring. The mixture was stirred for another $2 \mathrm{~h}$ to give rise to white precipitates. The obtained solid product was filtered, washed with deionized water and ethanol, and then dried in air. The dried sample was calcined at $550{ }^{\circ} \mathrm{C}$ for $1 \mathrm{~h}$ in $\mathrm{N}_{2}$ and followed by another $3 \mathrm{~h}$ in air to remove the organic template.

\section{Synthesis of 2-Cl-BT-MCM-41}

The MCM-41 was functionalized with 3aminopropyltriethoxysilane in the toluene solution (refluxing for $24 \mathrm{~h}$ ) according to the conventional procedure (19). After cooling, the modified mesoporous material was filtered out and washed several times with small portions of toluene and $i$-propanol to remove an excess of the modifier and possible products of hydrolysis. Finally, the modified mesoporous material was dried overnight in an oven at $95-100{ }^{\circ} \mathrm{C}$ under vacuum. The resulting material was designated as $\mathrm{MCM}-41-\mathrm{NH}_{2}$.

2-Cl-BT-MCM-41 was synthesized via a reaction between aminopropyl-functionalized mesoporous silica $\left(\mathrm{MCM}-41-\mathrm{NH}_{2}\right)$ and benzoyl isothiocyanate, which normally proceeds completely. A typical procedure included reaction of $9.04 \times 10^{-6} \mathrm{mmol}(2$ g) of aminopropylsilica with $0.50 \mathrm{~mL}$ of 2chlorobenzoyl isothiocyanate ( $25 \%$ excess) in toluene. The resulting solid was filtered out, washed with $50 \mathrm{~mL}$ of toluene and $50 \mathrm{~mL}$ of $i$ propanol, and dried under vacuum for $5 \mathrm{~h}$ at 90 ${ }^{\circ} \mathrm{C}$. The final sample with the attached thiourea functionality had a light yellow color. Schematic illustration of the surface present in the 2-Cl-BTMCM-41 is given in Figure 1.

\section{Characterization}

X-ray diffraction pattern was recorded on a Bruker D8 using $\mathrm{CuK}_{\mathrm{a}}$ radiation, with the diffraction angle (2O) at range of $10-80^{\circ}$. FT-IR spectrum was recorded on Perkin Elmer FT-
IR/FIR/NIR spectrometer Frontier ATR system. SEM measurements were recorded with a Jeol Sem-7100-EDX computer controlled digital model device. BET analyzes were done with Quantachrome Quadrasorb SI device. Absorbance measurements were performed with $\mathrm{Pg}$ Instruments UV-Visible spectrometer.

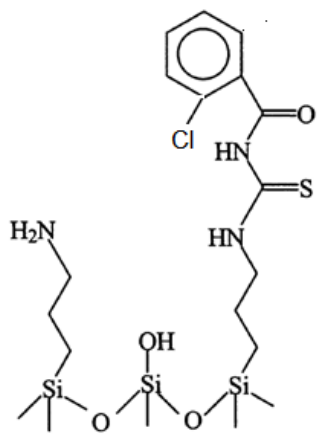

Figure: 1. Schematic illustration of the surface present in the 2-Cl-BT-MCM-41

\section{Ibuprofen loading and release studies}

Drug loading studies were carried out via supercritical carbon dioxide $\left(\mathrm{scCO}_{2}\right)$ system. $1 \mathrm{~g}$ of 2-Cl-BT-MCM-41 and $0.8 \mathrm{~g}$ ibuprofen were dissolved in $15 \mathrm{~mL}$ of ethanol and they were charged to the $\mathrm{ScCO}_{2}$ reaction unit at $40{ }^{\circ} \mathrm{C}$ and allowed to stand at 200 bar $\mathrm{CO}_{2}$ for 2 hours. The reaction unit's valve was opened to reduce the $\mathrm{CO}_{2}$ pressure and the drug loaded nanostructures were obtained.

The in vitro drug delivery was performed by soaking the sample powder into a simulated body fluid, SBF, at $37^{\circ} \mathrm{C}$ and at $\mathrm{pH}$ of 7.4 , maintaining the ratio $\mathrm{SBF}$ volume $(\mathrm{mL})$ per adsorbed ibuprofen mass (mg) equal to $0.8: 1$. Continuous magnetic stirring was maintained during the delivery to avoid limitation of the delivery rate by external diffusion constraints. The delivered ibuprofen concentration was monitored by UV spectrophotometry at $273 \mathrm{~nm}$.

\section{RESULTS AND DISCUSSION}

\section{FT-IR studies of MCM-41, MCM-41- $\mathrm{NH}_{2}$ and 2-Cl-BT-MCM-41}

FT-IR spectra of the MCM-41, MCM-41- $\mathrm{NH}_{2}$ and 2-Cl-BT-MCM-41 are given in Figure 2a-c. Asymmetric vibration band of the $\mathrm{O}-\mathrm{H}$ group is observed at $3356 \mathrm{~cm}^{-1}$. The stretching and bending vibration bands of $\mathrm{Si}-\mathrm{O}$ are observed at $1064 \mathrm{~cm}^{-1}$ and $801 \mathrm{~cm}^{-1}$, respectively. The results indicate the accuracy of the proposed MCM-41 structure. In the FT-IR spectrum of the MCM-41$\mathrm{NH}_{2}, \mathrm{~N}-\mathrm{H}$ vibration peaks were observed at 2850$2920 \mathrm{~cm}^{-1}$. In the FT-IR spectrum of the 2-Cl-BT$\mathrm{MCM}-41$, aromatic $\mathrm{C}-\mathrm{H}$ vibrations were observed at $3000 \mathrm{~cm}^{-1}$. $\mathrm{C}=\mathrm{O}$ vibrations also observed at $1700 \mathrm{~cm}^{-1}$. FT-IR spectra confirm that the proposed structures are formed. 


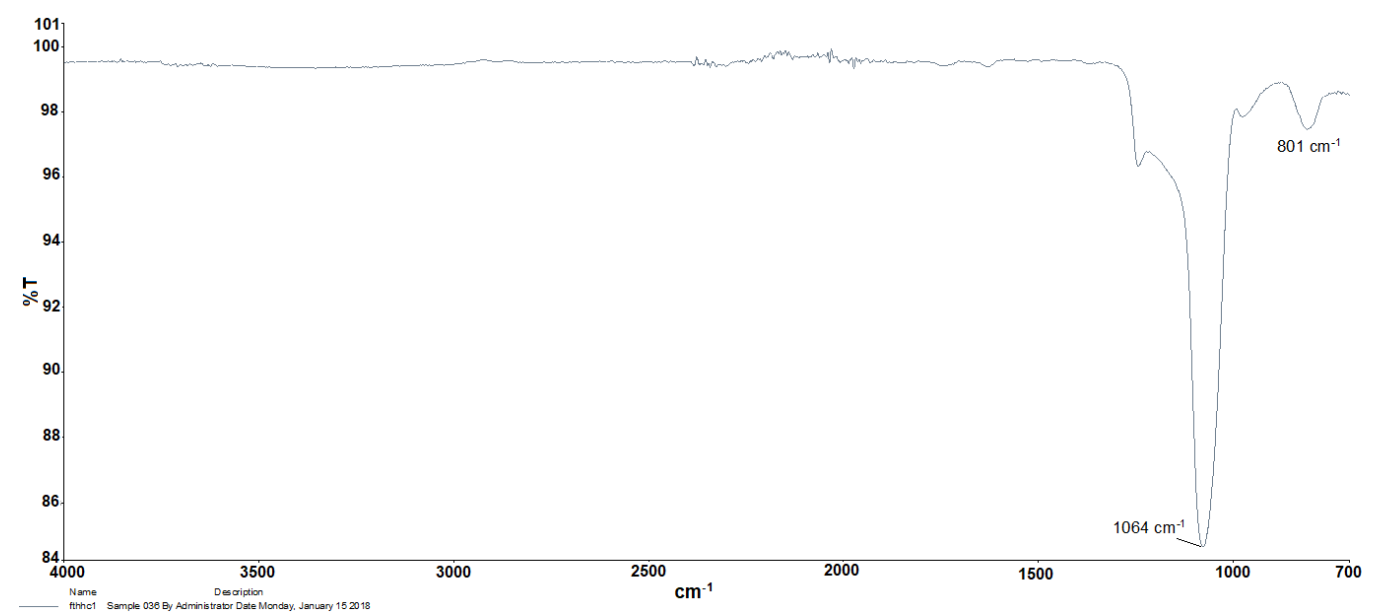

Figure 2: FT-IR spectrum of MCM-41.

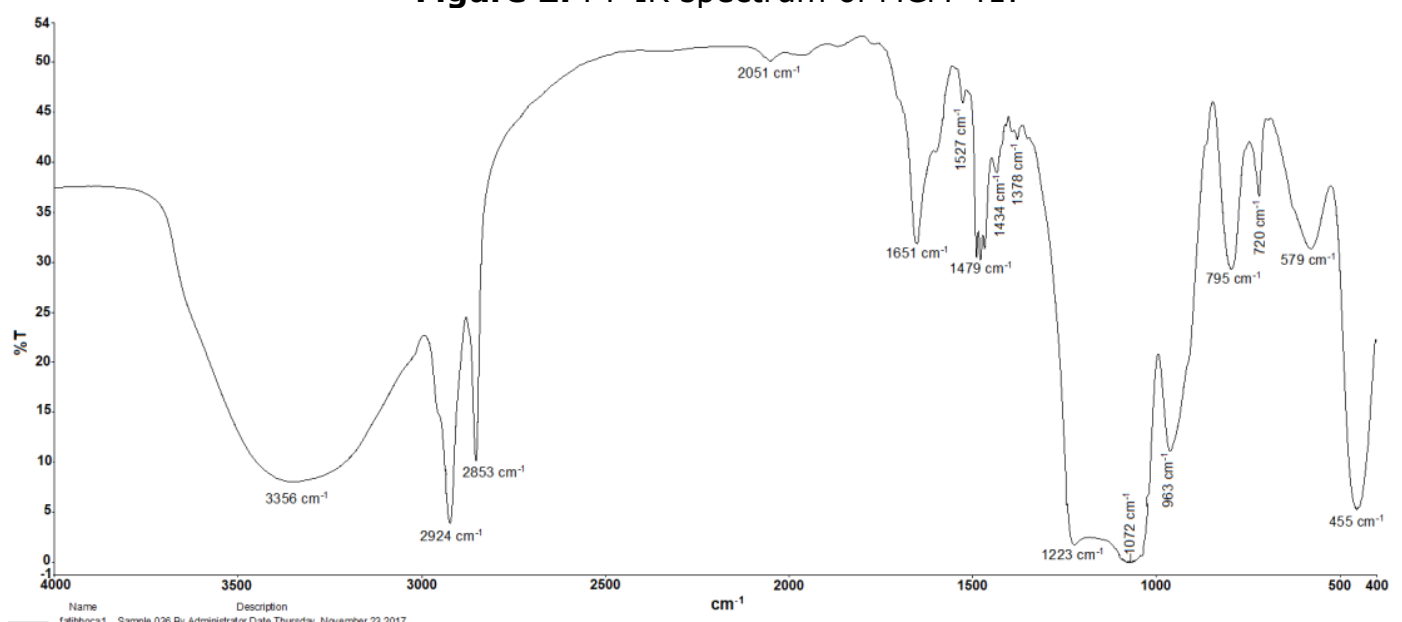

Figure 3: FT-IR spectrum of $\mathrm{MCM}-41-\mathrm{NH}_{2}$.

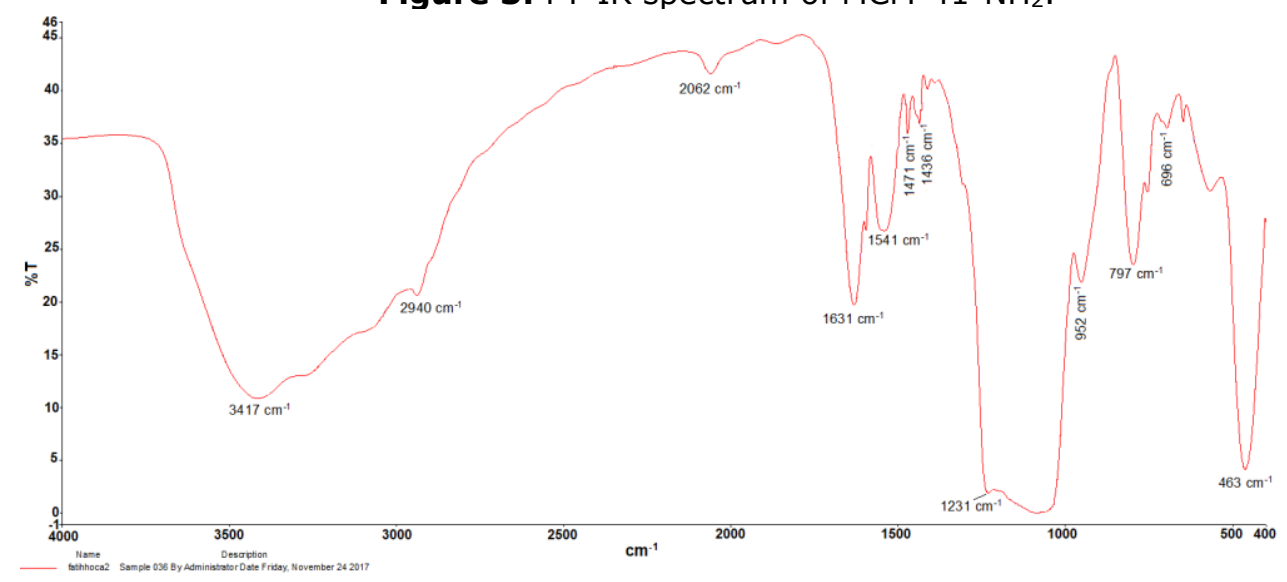

Figure 4: FT-IR spectrum of 2-Cl-BT-MCM-41.

\section{XRD studies}

The XRD pattern and low angle XRD patterns of 2-Cl-BT-MCM-41 are given in Figure 5a-b. The (100) and (110) reflections observed at low angle XRD show the mesoporous $\mathrm{SiO}_{2}$ structure.
The broad band observed in the XRD powder pattern at 2 theta $=20^{\circ}$ belongs to amorphous $\mathrm{SiO}_{2}$. XRD results show that mesoporous 2-Cl-BTMCM-41 has been successfully synthesized. 

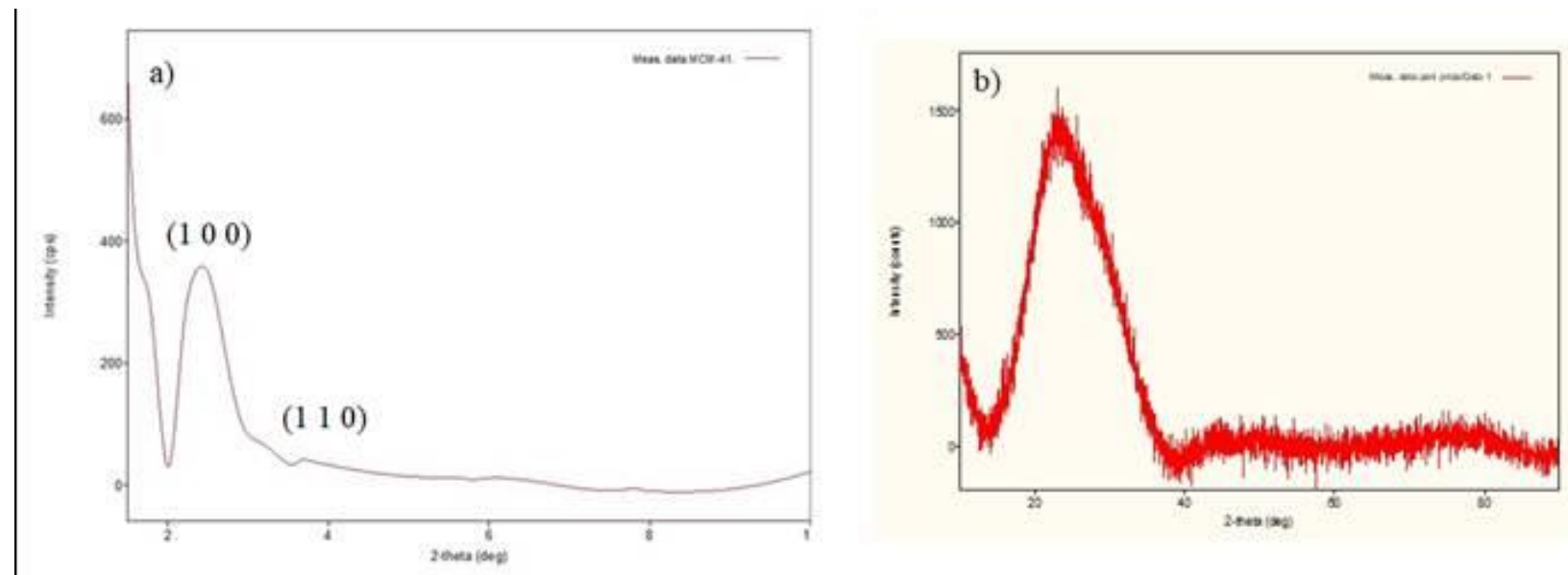

Figure 5: XRD powder patterns of MCM-41 a) Low angle XRD pattern, b) XRD pattern.

\section{SEM studies}

Surface investigations of the prepared 2-Cl-BT-MCM-41 were carried out by FE-SEM. SEM micrographs are given in Figure 6. SEM images showed non-uniform spheres at a range of 250-305 nm.
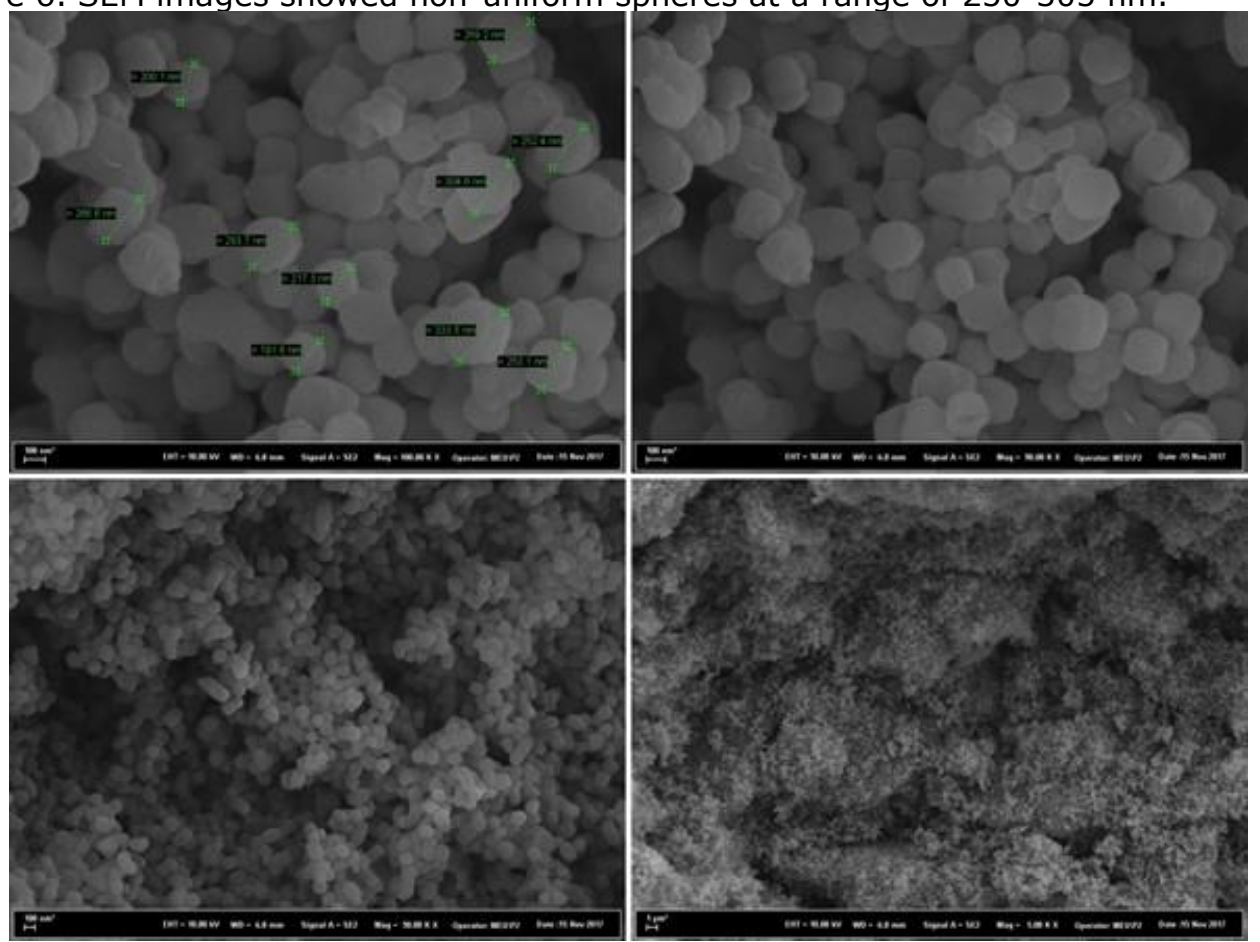

Figure 6: SEM images of 2-Cl-BT-MCM-41.

\section{In vitro Ibuprofen Releasing Studies}

To examine the drug release profiles, the cumulative percentages of drug release were plotted against time. The release behavior of ibuprofen from 2-Cl-BT-MCM-41was investigated in the PBS solution for 66 hours. Release of ibuprofen from 2-Cl-BT-MCM-41 in PBS solution
$\left(\mathrm{pH}=7.4 ; 37^{\circ} \mathrm{C}\right)$ is given in Figure 7 . Wave swing is observed in the drug release profiles. This behavior is due to unbalanced distribution of drug molecules from layered matrices, diffusibility, number of layers and thickness, and fluctuations in drug release are observed (20). 


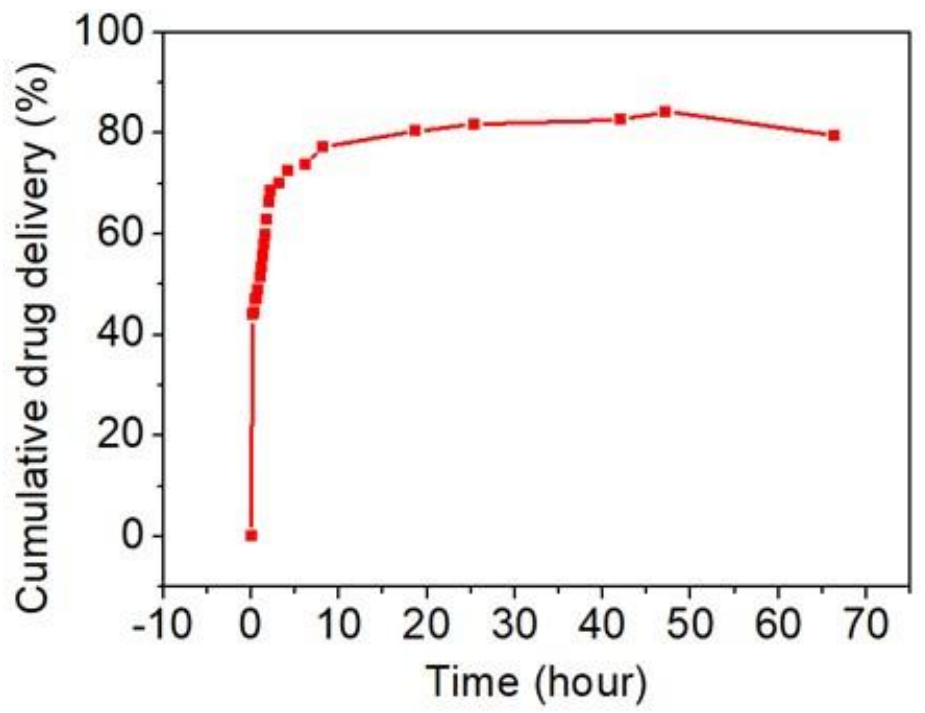

Figure 7: Release of ibuprofen from 2-Cl-BT-MCM-41in PBS solution $\left(\mathrm{pH}=7.4 ; 37^{\circ} \mathrm{C}\right)$.

\section{BET studies}

For the prepared MCM-41, the BET surface area was calculated as $1506 \mathrm{~m}^{2} / \mathrm{g}$ and is compatible with the $>1000 \mathrm{~m}^{2} / \mathrm{g}$ value given in the literature. In addition, the pore diameter of MCM-41 was

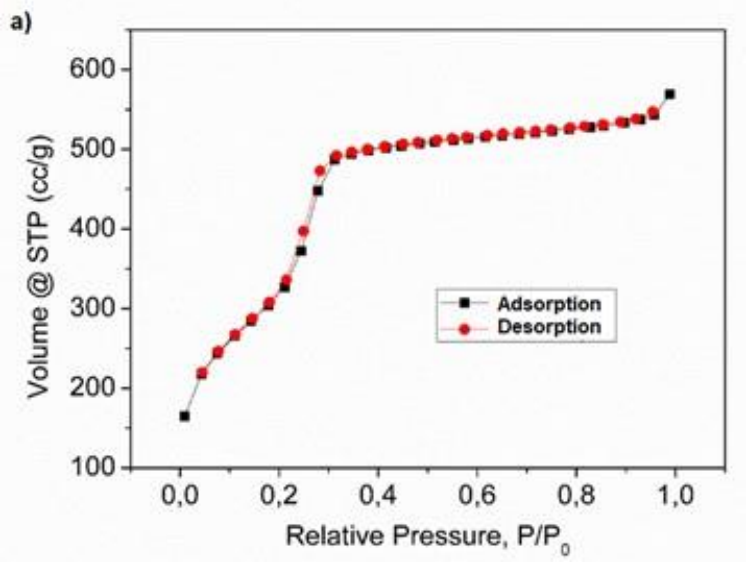

found to be $3.61 \mathrm{~nm}$ according to the literature. The surface area of 2-Cl-BT-MCM-41 was found to be $306 \mathrm{~m}^{2} / \mathrm{g}$. Nitrogen adsorption (black) desorption isotherms of MCM-41 and 2-Cl-BTMCM-41are given in $8 a-b$.

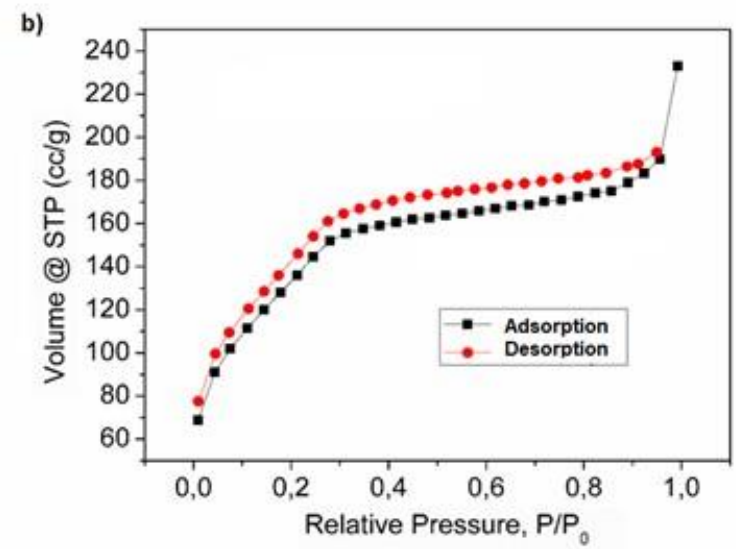

Figure 8: Nitrogen adsorption (black) desorption isotherms of a) MCM-41 and b) 2-Cl-BT-MCM-41.

\section{CONCLUSION}

In this study, mesoporous 2-Cl-BT-MCM-41was synthesized by precipitation method. 2-Cl-BTMCM-41 was characterized via XRD, FT-IR, SEM and BET techniques. FT-IR spectrum of the 2-ClBT-MCM-41, the aromatic vibrations $(\gamma \mathrm{C}-\mathrm{H})$ were observed at $3000 \mathrm{~cm}^{-1}$. The carbon-oxygen vibrations $(\gamma \mathrm{C}=0)$ were also observed at $1700 \mathrm{~cm}^{-}$ 1 . The (100) and (110) reflections observed at low angle XRD show the mesoporous $\mathrm{SiO}_{2}$ structure. The characterization results confirm that the proposed structures are formed. The surface morphology of the particles was determined by SEM micrographs. The particule size of nonuniform spheres were observed at a range of 250$305 \mathrm{~nm}$. BET surface areas were calculated as $1506 \mathrm{~m}^{2} / \mathrm{g}$ for MCM-41 and $306 \mathrm{~m}^{2} / \mathrm{g} \mathrm{2-Cl-BT-}$ MCM-41, respectively. BET surface area is given as $>1000 \mathrm{~m}^{2} / \mathrm{g}$ value in the literature. Our BET results are compatible with the literature. Ibuprofen was loaded using supercritical carbon dioxide $\left(\mathrm{sC}-\mathrm{CO}_{2}\right)$ environment. The absorption and releasing studies of ibuprofen were carried out in simulated body fluid. The result revealed that high adsorption capacity for drug with 2-ClBT-MCM-41 and slower drug release rate was achieved. MCM 41 and 2-Cl-BT-MCM-41 bind with ibuprofen over weak hydrogen interactions. This causes slow releasing.

\section{ACKNOWLEDGEMENTS}

This study was supported by Mehmet Akif Ersoy University Scientific Research Projects Council (Project Number 0434-Yl-17).

\section{REFERENCES}

1. Vallet-Regi M. Ramila A. Del Real RP. PérezPariente J. A new property of MCM-41: drug delivery system. Chem.Mater. 2001; 13(2):308-11. 
2. Wang, S. Ordered mesoporous materials for drug delivery. Micropor. Mesopor. Mat. 2009; 117(1-2): 1-9.

3. Zeng W. Qian XF. Yin J. Zhu ZK. The drug delivery system of MCM-41 materials via cocondensation synthesis. Mater. Chem. Phys. 2006; 97(2-3): 437-41.

4. Sousa A. Souza KC. Sousa EMB. Mesoporous silica/apatite nanocomposite: special synthesis route to control local drug delivery. Acta. Biomater. 2008; 4(3): 671-9.

5. Qu F. Zhu G. Huang S. Li S. Sun J. Zhang D. Qiu S.Controlled release of Captopril by regulating the pore size and morphology of ordered mesoporous silica. Micropor. Mesopor. Mat. 2006; 92(1-3): 1-9.

6. Mal NK. Fujiwara M. Tanaka Y. Taguchi T. Matsukata M. Photo-switched storage and release of guest molecules in the pore void of coumarinmodified MCM-41. Chem. Mater. 2003; 15(17): 3385-34.

7. Aiello R. Cavallaro G. Giammona G. Pasqua L. Pierro P. Testa F. Mesoporous silicate as matrix for drug delivery systems of non-steroidal antinflammatory drugs. In Studies in Surface Science and Catalysis Elsevier. 2002; 142:116572.

8. Cavallaro G. Pierro P. Palumbo FS. Testa F. Pasqua L. Aiello R. Drug delivery devices based on mesoporous silicate. Drug. Deliv. 2004; 11(1):41-6.

9. Vallet-Regí M. Balas F. Arcos D. Mesoporous materials for drug delivery. Angew. Chem.Int. Edit 2007; 46(40):7548-58.

10. Xu W. Xu Y. Wu D. Sun Y. Ibuprofen delivery systems based on monodispersed spherical MCM41 materials. In Studies in Surface Science and Catalysis Elsevier. 2007; 170:861-5.

11. Manzano M. Aina V. Arean CO. Balas F. Cauda V. Colilla M. Vallet-Regi M. Studies on MCM-41 mesoporous silica for drug delivery: effect of particle morphology and amine functionalization. Chem. Eng. J. 2008; 137(1):30-7.
12. Carino IS. Pasqua L. Testa F. Aiello R. Puoci F. Iemma F. Picci N. Silica-based mesoporous materials as drug delivery system for methotrexate release. Drug. Deliv. 2007; 14(8):491-5.

13. Nunes CD. Vaz PD. Fernandes AC. Ferreira P. Romao CC. Calhorda MJ. Loading and delivery of sertraline using inorganic micro and mesoporous materials. Eur. J. Pharm. Biopharm. 2007; 66(3):357-65.

14. Vallet-Regi M. Ramila A. Del Real RP. PérezPariente J. A new property of MCM-41: drug delivery system. Chem. Mater. 2001; 13(2):30811 .

15. Popova M. Szegedi A. Mavrodinova V. Tušar NN. Mihály J. Klébert S. Yoncheva K. Preparation of resveratrol-loaded nanoporous silica materials with different structures. J. Solid State Chem. $2014 ; 219: 37-42$.

16. Horcajada P. Ramila A. Perez-Pariente J. Vallet-Regı M. Influence of pore size of MCM-41 matrices on drug delivery rate. Micropor. Mesopor. Mat. 2004; 68(1-3):105-9.

17. Rámila A. Munoz B. Pérez-Pariente J. ValletRegí M. Mesoporous MCM-41 as drug host system. J. Sol-Gel Sci. Tech. 2003; 26(13):1199-202.

18. Vallet-Regí M. Balas F. Arcos D. Mesoporous materials for drug delivery. Angew. Chem.Int. Edit. 2007; 46(40):7548-58.

19. Antochshuk V. Jaroniec M. Adsorption, thermogravimetric, and NMR studies of FSM-16 material functionalized with alkylmonochlorosilanes. J. Phys. Chem. B. 1999; 103(30):6252-61.

20. Dash S. Murthy PN. Nath L. Chowdhury P. Kinetic modeling on drug release from controlled drug delivery systems. Acta. Pol. Pharm. 2010;67(3):217-23. 